\title{
Synthesis of Methylated Anthranilate Derivatives Using Engineered Strains of Escherichia colis
}

\author{
Hye Lim Lee $^{1 \dagger}$, Song-Yi Kim ${ }^{1 \dagger}$, Eun Ji Kim ${ }^{1}$, Da Ye Han ${ }^{1}$, Bong-Gyu Kim², and Joong-Hoon Ahn ${ }^{1 *}$ \\ ${ }^{1}$ Department of Integrative Bioscience and Biotechnology, Bio/Molecular Informatics Center, Konkuk University, Seoul 05029, Republic of Korea \\ ${ }^{2}$ Department of Forest Resources, Gyeongnam National University of Science and Technology, Jinju 52725, Republic of Korea
}

\author{
Received: April 15, 2019 \\ Revised: May 27, 2019 \\ Accepted: June 3, 2019 \\ First published online \\ June 4, 2019 \\ ${ }^{*}$ Corresponding author \\ Phone: +82-2-45-3764; \\ Fax: +82-2-3437-6106; \\ E-mail: jhahn@konkuk.ac.kr \\ ${ }^{\dagger}$ These authors contributed \\ equally to this work. \\ $S$ upplementary data for this \\ paper are available on-line only at \\ http://jmb.or.kr. \\ pISSN 1017-7825, eISSN 1738-8872 \\ Copyright@ 2019 by \\ The Korean Society for Microbiology \\ and Biotechnology
}

\begin{abstract}
Anthranilate derivatives have been used as flavoring and fragrant agents for a long time. Recently, these compounds are gaining attention due to new biological functions including antinociceptive and analgesic activities. Three anthranilate derivatives, $N$-methylanthranilate, methyl anthranilate, and methyl $N$-methylanthranilate were synthesized using metabolically engineered stains of Escherichia coli. NMT encoding $N$-methyltransferase from Ruta graveolens, AMAT encoding anthraniloyl-coenzyme A (CoA):methanol acyltransferase from Vitis labrusca, and $p q s A$ encoding anthranilate coenzyme A ligase from Pseudomonas aeruginosa were cloned and $E$. coli strains harboring these genes were used to synthesize the three desired compounds. E. coli mutants (metJ, trpD, tyrR mutants), which provide more anthranilate and/or S-adenosyl methionine, were used to increase the production of the synthesized compounds. MS/MS analysis was used to determine the structure of the products. Approximately, $185.3 \mu \mathrm{M}$ $N$-methylanthranilate and $95.2 \mu \mathrm{M}$ methyl $N$-methylanthranilate were synthesized. This is the first report about the synthesis of anthranilate derivatives in E. coli.
\end{abstract}

Keywords: Anthranilate, $N$-methyltransferase, anthraniloyl-coenzyme A (CoA):methanol acyltransferase, metabolic engineering

\section{Introduction}

Anthranilate (2-aminobenzoic acid) has long been used for the synthesis of diverse compounds including perfumes, pharmaceuticals, and insect repellents [1]. Biologically, it is an intermediate of tryptophan biosynthesis [2] and alkaloids [3].

Alkylated $N$-methylanthranilates have been used as flavoring and fragrant agents [4, 5]. Methyl $N$-methylanthranilate, a flavoring compound, was discovered from a variety of grape and Choisya ternata Kunth; it has been used in the cosmetic industry. Ethyl $\mathrm{N}$-methylanthranilate also has similar properties. Alkylate anthranilates such as methyl anthranilate and ethyl anthranilate are natural compounds that are responsible for flavor and fragrance [6]. They are also known to be insect repellent and to have antipathogenic effects $[7,8]$. Additional biological activities of these anthranilate derivatives have been found; Methyl $\mathrm{N}$-methylanthranilate and isopropyl $\mathrm{N}$-methylanthranilate have shown antidepressant-like activity [9]. Isopropyl $\mathrm{N}$ methylanthranilate has antinociceptive activity [5]. A recent report speculated that methyl $\mathrm{N}$-methylanthranilate has analgesic activity [10]. Usually, alkylated $N$-methylanthranilates have a methyl or ethyl group attached to anthranilate through $\mathrm{N}$-methylation and/or ester formation with a carboxyl group.

Methyl anthranilate is one of the ingredients of Concord grape and contributes to the flavor of wines produced from this variety of grapes [11]. In addition, methyl anthranilate has been used as a bird repellent for crop protection [12,13].

Biological synthesis of these anthranilates has been studied in some plants. N-Methylanthranilate is synthesized from anthranilate by an enzyme anthranilate $N$-methyl transferase (NMT), which uses S-adenosyl methionine (SAM) as a methyl group donor. NMT from Ruta graveolens has been cloned [14]. Moreover, biosynthesis of methyl anthranilate was studied in Vitis labrusca and anthraniloylcoenzyme A (CoA):methanol acyltransferase (AMAT), 


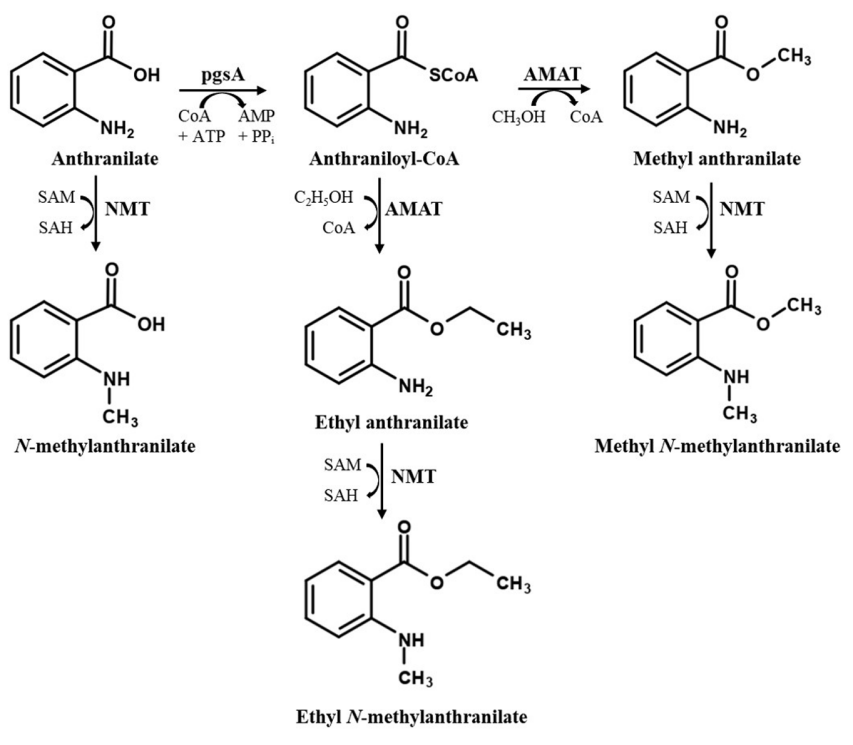

Fig. 1. Scheme for the synthesis of anthranilate derivatives $(N-$ methyl anthranilate, methyl anthranilate, ethyl anthranilate, methyl $N$-methylanthranilate, and ethyl $N$-methylanthranilate).

which is responsible for the formation of methyl anthranilate, has also been cloned [15]. AMAT uses diverse alcohols as an alkyl group donor and anthraniloyl-CoA as an alkyl group acceptor. The attachment of coenzyme A (CoA) to anthranilate is mediated by pqs A from Pseudomonas aeruginosa [16]. The combination of these genes would make it possible to synthesize various anthranilate derivates.

Escherichia coli has been widely used to synthesize natural compounds [17] because precursors for the synthesis of various compounds and cofactors such as SAM, CoA, and nucleotide sugars can be easily provided in the medium, and manipulation of metabolic pathways is straightforward. E. coli synthesizes anthranilate, which can be used for the synthesis of various anthranilate derivatives (Fig. 1). By introducing NMT, AMAT, and pqsA into E. coli and engineering $E$. coli metabolic pathways, we synthesized three anthranilate derivates.

\section{Materials and Methods}

\section{Nucleic Acid Manipulation}

Ruta graveolens and Concord grape (Vitis labrusca) were purchased from a local market and total RNA was isolated using a Plant Total RNA Isolation Kit (Qiagen, US). The isolated RNA was used as template for reverse transcription-polymerase chain reaction (RT-PCR). Anthranilate $N$-methyltransferase (NMT) and anthranilate methyltransferase (AMAT) were cloned from $R$. graveolens and $V$. labrusca, respectively. Primers were designed based on the published nucleotide sequences (NMT, DQ884932.1;
AMAT, AY705388). Primer sequences for NMT were 5'-ATGGAT CCGATGGGTTCTTTATCGGAATCC- $3^{\prime}$ as a forward primer and 5'-ATGCGGCCGCCTACTTGAAGAACTCCATGA-3' as a reverse primer (restriction enzyme sites for BamHI at the forward primer and NotI at the reverse primer are underlined). The PCR product was sequenced and subcloned into the corresponding site of pETDuet1 vector (pE-NMT). 5'-AAGTCGACATGGCATCATCGTCGT CTCC-3' (Sall site is underlined.) and 5'-AAGCGGCCGCTTAGG GCATGGATGTAATTAACAGC-3' (NotI site is underlined.) were used as forward and reverse primers for $A M A T$, respectively. AMAT gene was subcloned into SalI/NotI site of pCDFduet vector (pC-AMAT). trpEG from E. coli was cloned using PCR. 5'ATGGATCCCATGCAAACACAAAAACCGACT-3' and 5'AT드 TCGAGTTACAGAATCGGTTGCAGCGTG-3' were used as the forward and the reverse primers, respectively. BamHI and XhoI site was introduced into the forward primer and the reverse primer, respectively. The resulting PCR product was sequenced and subcloned into the second cloning site (BglII/XhoI) of $\mathrm{pE}$ NMT to produce $\mathrm{pE}-\mathrm{NMT}$-trpEG.

PqsA gene was cloned by PCR using genomic DNA of Pseudomonas aeruginosa as a template. Following primers were used; 5'-ATCATATGTCCACATTGGCCAACCTGACCG-3' (NdeI site is underlined) and $5^{\prime}$ CATCTCGAGTCAACATGCCCGTTC CTCCGG3' (XhoI site is underlined). The resulting PCR product was subcloned into NdeI/Xhol site of pC-AMAT and the resulting construct was named pC-AMAT-pqsA.

\section{Production of Methylated Anthranilates}

$N$-Methylanthranilate was synthesized using E. coli harboring either pE-NMT or pE-NMT-trpEG. E. coli transformants were grown and gene expression was induced as described in Song et al. [18]. Cells were harvested by centrifugation and resuspended in M9 medium containing $100 \mu \mathrm{M}$ anthranilate, $50 \mu \mathrm{g} / \mathrm{ml}$ ampicillin, and $1 \mathrm{mM}$ IPTG. The reaction was carried out for $24 \mathrm{~h}$. Synthesis of $\mathrm{N}$-methylanthranilate using various E. coli strains was carried out the same as above except that anthranilate was not added.

Methyl anthranilate was synthesized using E. coli harboring pCAMAT-pqsA. After harvesting cells, they were resuspended using M9 medium containing $100 \mu \mathrm{M}$ anthranilate, $5 \%$ alcohol (methanol, ethanol, isopropanol, or butanol), $50 \mu \mathrm{g} / \mathrm{ml}$ spectinomycin, and $1 \mathrm{mM}$ IPTG. Culture filtrate was extracted with ethyl acetate and the organic layer was dried and dissolved in DMSO. The dissolved sample was analyzed using high-performance liquid chromatography (HPLC) [19]. N-Methylanthranilate was detected at $250 \mathrm{~nm}$. Methyl anthranilate, ethyl anthranilate, and methyl $\mathrm{N}$ methylanthranilate were detected at $354 \mathrm{~nm}$. Standard deviation and mean were calculated based on triplicate experiments. The structure of $\mathrm{N}$-methylanthranilate was determined using nuclear magnetic resonance spectroscopy (NMR) [20]; ${ }^{1} \mathrm{H} \mathrm{NMR}(400 \mathrm{MHz}$, DMSO-d6): $\delta 7.77(\mathrm{dd}, \mathrm{J}=7.9,1.5 \mathrm{~Hz}, 1 \mathrm{H}), 7.37(\mathrm{td}, \mathrm{J}=7.8,1.6 \mathrm{~Hz}$, $1 \mathrm{H}), 6.67(\mathrm{~d}, \mathrm{~J}=8.4 \mathrm{~Hz}, 1 \mathrm{H}), 6.54(\mathrm{t}, \mathrm{J}=7.4 \mathrm{~Hz}, 1 \mathrm{H}), 2.83(\mathrm{~s}, 3 \mathrm{H})$. MS/MS analysis was carried out as previously described [21]. Mass spectra were acquired in the positive ionization mode. 
Synthesis of anthranilate from glucose was carried out as described previously [18]. For the synthesis of alkyl anthranilate, alcohol was provided at the final concentration of $5 \%(\mathrm{v} / \mathrm{v})$. And, the cells were grown at $30^{\circ} \mathrm{C}$ at $180 \mathrm{rpm}$ for $24 \mathrm{~h}$.

\section{Results and Discussion}

\section{Synthesis of $\mathrm{N}$-Methylanthranilate}

$\mathrm{N}$-methylanthranilate is synthesized from anthranilate in a reaction catalyzed by NMT. To synthesize $N$ methylanthranilate, E. coli strain B1 was fed with anthranilate and the culture filtrate was analyzed. As shown in Fig. 2, the reaction product had the same retention time as $N$ methylanthranilate. The structure of this reaction product was analyzed by NMR and it was confirmed to be $N$ methylanthranilate.

Anthranilate is an intermediate for the synthesis of tryptophan; however, it is also a substrate for NMT. The production of $\mathrm{N}$-methylanthranilate can be increased by engineering anthranilate production. We examined the production of $\mathrm{N}$-methylanthranilate from glucose. As expected, the strain B1 synthesized approximately $1.6 \mathrm{mg} / 1$ $N$-methylanthranilate without feeding anthranilate. In order to increase the production, we next overexpressed trpEG gene encoding anthranilate synthase that catalyzes

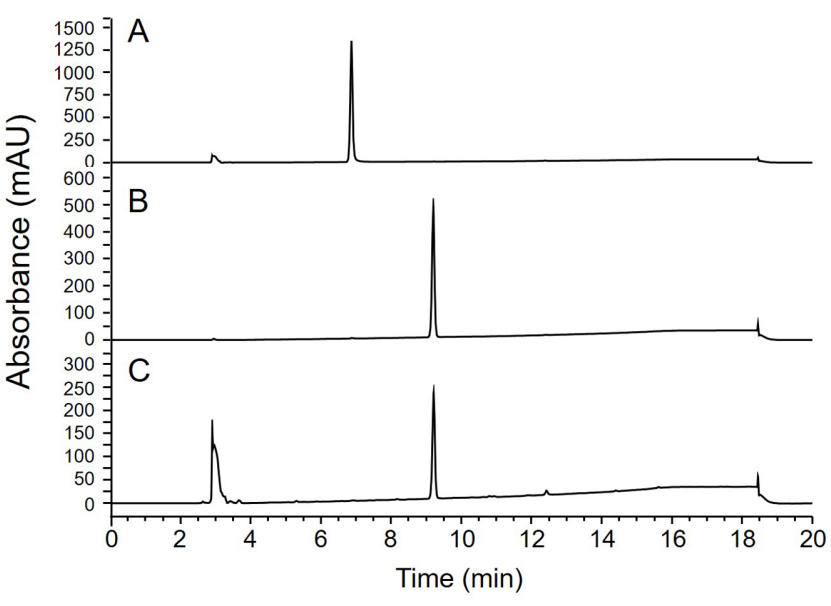

Fig. 2. Synthesis of $N$-methylanthranilate using E. coli harboring NMT.

A, anthranilate standard; B, N-methylanthranilate standard; C, the reaction product from $E$. coli harboring NMT.

the conversion of chorismate to anthranilate. The resulting strain B2 produced $16.6 \mathrm{mg} / 1 \mathrm{~N}$-methylanthranilate. Then, we used E. coli tyrR and trpD double mutant (B-DR in Table 1). TyrR is a negative regulator of transcription and its deletion resulted in the activation of the first step of shikimate pathway [22]. $\operatorname{Trp} D$ encodes anthranilate

Table 1. Plasmids and strains used in the present study.

\begin{tabular}{|c|c|c|}
\hline Plasmids or E. coli strains & Relevant properties or genetic markers & Source \\
\hline \multicolumn{3}{|l|}{ Plasmids } \\
\hline pETDuet & f1 ori, $\mathrm{Amp}^{\mathrm{r}}$ & Novagen \\
\hline pE-NMT & pETduet + NMT from Ruta graveolens & \\
\hline pE-trpEG & pETduet + trpEG from Escherichia coli & This study \\
\hline pE-trpEG-NMT & pETduet + trpEG from Escherichia coli + NMT from $R$. graveolens & This study \\
\hline \multicolumn{3}{|l|}{ Strains } \\
\hline BL21 (DE3) & $\mathrm{F}^{-} \operatorname{omp} T h s d S_{B}\left(\mathrm{r}_{\mathrm{B}}^{-} \mathrm{m}_{\mathrm{B}}^{-}\right)$gal dcm lon (DE3) & \\
\hline B-DR & BL21(DE3) $\Delta t r p D / \Delta t y r R$ & This study \\
\hline B-DRM & BL21(DE3) $\Delta t r p D / \Delta t y r R / \Delta M e t J$ & This study \\
\hline B2 & BL21 (DE3) harboring pE-trpEG-NMT & This study \\
\hline B3 & BL21 (DE3) harboring pE-trpEG and pC-AMAT-pqsA & This study \\
\hline B4 & BL21 (DE3) harboring pC-AMAT-pqsA & This study \\
\hline B-DR1 & B-DR harboring pE-trpEG-NMT & This study \\
\hline B-DRM1 & B-DRM harboring pE-trpEG-NMT & This study \\
\hline B-MMA1 & E. coli BL21 harboring pE-trpEG-NMT and pC-AMAT-pqsA & This study \\
\hline B-MMA2 & E. coli B-DR harboring pE-trpEG-NMT and pC-AMAT-pqsA & This study \\
\hline
\end{tabular}




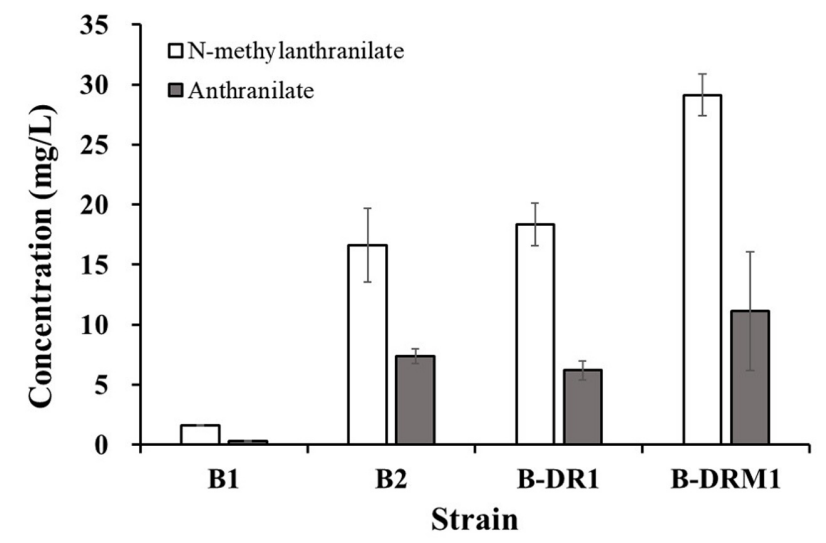

Fig. 3. Synthesis of $N$-methylanthranilate from glucose using various E. coli strains.

phosphoribosyl transferase, which converts anthranilate into $N$-(5'-phosphoribosyl)-anthranilate [1]. The strain B-DR1 synthesized $18.4 \mathrm{mg} / 1 \mathrm{~N}$-methylanthranilate. Furthermore, we engineered the strain for the production of methyl group donor. SAM serves as a methyl group donor. MetJ is a repressor of SAM synthesis and it inhibits the production of SAM using a feedback mechanism [23]. Therefore, we made MetJ deletion mutant in order to make more SAM available for the synthesis of $\mathrm{N}$-methylanthranilate. (Table 1 ). The resultant triple mutant strain (B-DRM1, Table 1) produced approximately $29.1 \mathrm{mg} / 1 \mathrm{~N}$-methylanthranilate (Fig. 3). We detected unreacted anthranilate in all the E. coli strain. Although both anthranilate and SAM are critical for $N$-methylanthranilate production, conversion of anthranilate into $N$-methylanthranilate was slower than the synthesis of anthranilate.

\section{Synthesis of Alkyl Anthranilate}

We tested the synthesis of alkyl anthranilates such as methyl anthranilate and ethyl anthranilate. This reaction is catalyzed by two enzymes. Anthranilate is converted into anthraniloyl-CoA by anthranilate-CoA ligase and then it is converted into alkyl anthranilate by AMAT. Which alkyl group is attached to anthranilate depends on the alcohol used. In order to supply more anthranilate, trpEG was overexpressed in E. coli. Three genes (trpEG, pqsA, and $A M A T)$ were introduced into $E$. coli and the transformants (B3) were grown in the presence of $2 \%$ of methanol, ethanol, propanol, isopropanol, or butanol. B3 fed with methanol, ethanol, or isopropanol showed a new peak in HPLC, which had a different retention time than that of anthranilate. Reaction products from methanol and ethanol

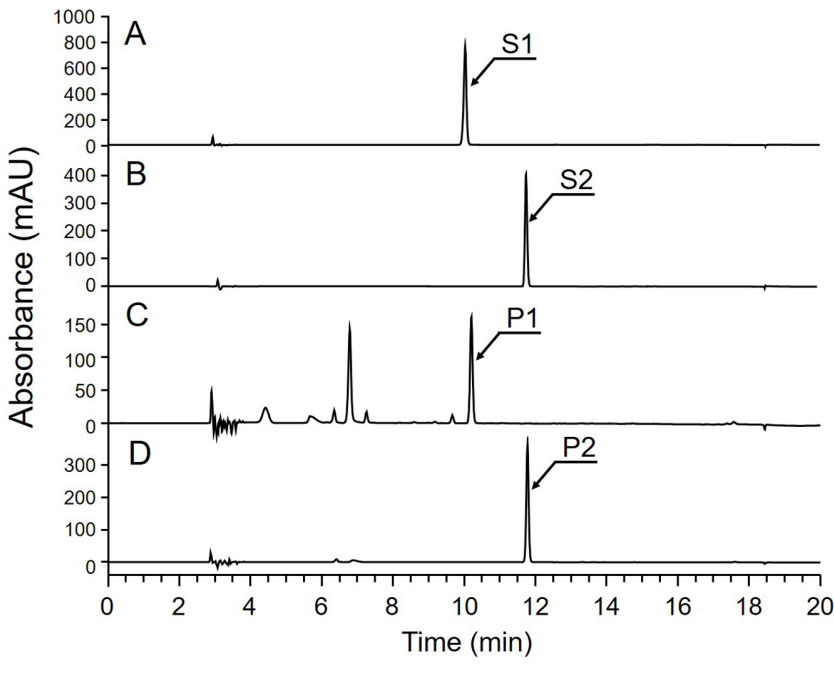

Fig. 4. Production of methyl anthranilate and ethyl anthranilate using E. coli strain B3.

A, methyl anthranilate standard (S1); B, ethyl anthranilate standard (S2); C, reaction product (P1) after feeding methanol; D, reaction product (P2) after feeding ethanol. mAU: milli absorption unit.

had the same HPLC retention time as that of the standards methyl anthranilate and ethyl anthranilate, respectively (Fig. 4). The molecular masses of the products from the reactions involving methanol and ethanol were 152.0698 and $166.0854 \mathrm{Da}$, respectively (Figs. S1 and S3). These agreed with the predicted molecular masses of methyl anthranilate and ethyl anthranilate, respectively. Furthermore, the two compounds synthesized when methanol or ethanol were fed showed the MS/MS fragmentation patterns matching with methyl anthranilate or ethyl anthranilate, respectively (Figs. S1 and S3). These results indicated that the strain B3 synthesized methyl anthranilate and ethyl anthranilate. Ethanol was the best substrate followed by methanol and isopropanol. Only a small amount of isopropanol reacted with anthranilate (data not shown). This result was in contrast with the enzymatic reaction result [15], in which isopropanol was the best followed by ethanol and methanol. This might be due to the different permeability of each alcohol into E. coli. Isopropanol was less permeable than the other two alcohols, which limited the supply of isopropanol for the synthesis of the isopropyl anthranilate.

\section{Synthesis of Methyl N-Methylanthranilate}

Next, we synthesized methyl $N$-methylanthranilate. Synthesis of methyl $\mathrm{N}$-methylanthranilate can be achieved 


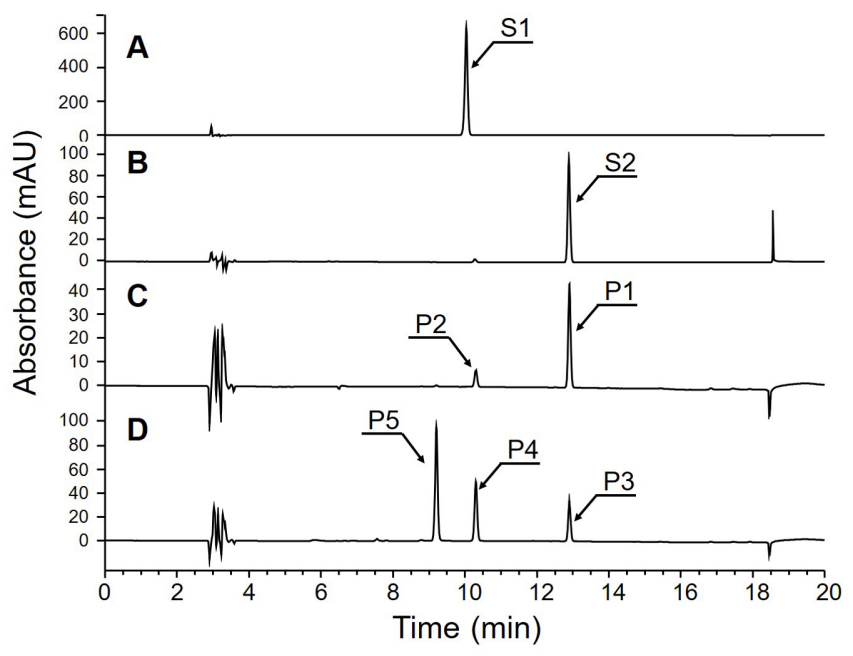

Fig. 5. Synthesis of methyl $N$-methylanthranilate in E. coli. A, standard methyl anthranilate; B, standard methyl $N$ methylanthranilate; $\mathrm{C}$, the reaction product from $\mathrm{B}, \mathrm{MMA} 1$; $\mathrm{D}$, the reaction product from $B, M M A 2$. $\mathrm{mAU}$ : milli absorption unit.

by two routes; the choice depends on the compound first synthesized, $N$-methylanthranilate or methyl anthranilate. Two constructs, pE-trpEG-NMT and pC-AMAT-pqsA were transformed into E. coli BL21 (B-MMA1) and B-DR (BMMA2). The synthesis of methyl $N$-methylanthranilate was examined in each transformant after feeding methanol. Both transformants successfully synthesized a new compound that had the same HPLC retention time as that of methyl $\mathrm{N}$-methylanthranilate (Fig. 5). The molecular mass of the synthesized compound was $166.0854 \mathrm{Da}$, which corresponds with that of methyl $\mathrm{N}$-methylanthranilate. Also, the MS/MS fragmentation pattern of the synthesized compound matched with that of methyl $N$-methylanthranilate (Fig. S2). Taken together, methyl $N$-methylanthranilate was successfully synthesized in both the E.coli strains. Moreover, when ethanol was fed to these strains, ethyl $\mathrm{N}$ methylanthranilate was synthesized (Fig. S4).

Notably, B-MMA1 synthesized more methyl $N$ methylanthranilate than B-MMA2. B-MMA2 accumulated unreacted products such as $N$-methylanthranilate and methyl anthranilate. B-MMA2 produced more anthranilate, which could be a substrate for either NMT or AMAT. The conversion to $\mathrm{N}$-methylanthranilate was faster than that to methyl anthranilate as shown in Fig. 5D. It was likely that methyl anthranilate was not converted into methyl $\mathrm{N}$-methylanthranilate and inhibited the conversion of $\mathrm{N}$-methylanthranilate into methyl $\mathrm{N}$-methylanthranilate.
In order to test this, methyl anthranilate was fed to E. coli cells harboring pE-NMT. We found that only $10 \%$ of methyl anthranilate was converted into methyl $\mathrm{N}$-methyl anthranilate (data not shown). On the other hand, when $N$-methylanthranilate along with methanol was fed to E. coli harboring pC-AMAT-pqsA, most of the $N$-methylanthranilate was converted into methyl $N$-methylanthranilate. These results indicated that the strain B-MMA1 synthesized more methyl $\mathrm{N}$-methylanthranilate; less synthesis of anthranilate in the strain B-MMA1 led to the conversion of the synthesized anthranilate into $N$-methylanthranilate or methyl anthranilate, and then these were converted to methyl $\mathrm{N}$-methylanthranilate, although some methyl anthranilate still remained.

We determined the optimal concentration of alcohol (methanol or ethanol) to maximize the synthesis of methyl $\mathrm{N}$-methylanthranilate and ethyl $\mathrm{N}$-methylanthranilate. We fed $1 \%, 3 \%, 5 \%, 8 \%$, or $10 \%$ alcohol along with $N$-methyl anthranilate to E. coli strain B4. The synthesis of alkyl Nmethylanthranilate increased until 5\% alcohol was added. At the concentrations higher than $8 \%$, the synthesis of alkyl $N$-methylanthranilate decreased. This might be due to the toxic effects of alcohol on E. coli.

To increase the synthesis of methyl N-methylanthranilate, we divided the whole synthesis pathway into two steps; we synthesized $N$-methylanthranilate using B-DRM1 (Table 1) in the first step and then the filtrate of this culture along with methanol was fed to E. coli strain B4 in the second step to synthesize methyl $N$-methylanthranilate from $\mathrm{N}$-methylanthranilate. Approximately $29.1 \mathrm{mg} / 1 \mathrm{~N}$ methylanthranilate (approximately $192.6 \mu \mathrm{M}$ ) was synthesized using B-DRM1. The resulting supernatant was mixed with E. coli BL21 harboring pC-AMAT-pqsA along with 5\% methanol or ethanol. All the $N$-methylanthranilate was converted into the corresponding alkyl N-methylanthranilate; however, approximately $95.2 \mu \mathrm{M}$ methyl $N$-methylanthranilate could be detected because methyl $N$-methylanthranilate is volatile.

We successfully synthesized anthranilate derivatives $(N$ methylanthranilate, methyl anthranilate, ethyl anthranilate, methyl $N$-methylanthranilate, and ethyl $N$-methylanthranilate) using engineered E. coli strains. Methyl (ethyl) anthranilate and methyl (ethyl) $\mathrm{N}$-methylanthranilate were volatile and the estimated final yields of these compounds were somewhat lower than their actual yields. This is the first report of the synthesis of these anthranilate derivatives in E. coli and opens a way to synthesize flavoring compounds using engineered $E$. coli strains. 


\section{Acknowledgments}

This work was funded by a grant from the NextGeneration BioGreen 21 Program (PJ01326001), Rural Development Administration, Republic of Korea.

\section{Conflict of Interest}

The authors have no financial conflicts of interest to declare.

\section{References}

1. Balderas-Hernández VE, Sabido-Ramos A, Silva P, CabreraValladares N, Hernández-Chávez G, Báez-Viveros JL, et al. 2009. Metabolic engineering for improving anthranilate synthesis from glucose in Escherichia coli. Microb. Cell Fact. 8: 19.

2. Radwanski ER, Last RL.1995. Tryptophan biosynthesis and metabolism: biochemical and molecular genetics. Plant Cell 7: 921-934.

3. Kutchan TM. 1995. Alkaloid biosynthesis-The basis for metabolic engineering of medicinal plants. Plant Cell 7: 1059-1070.

4. Pillet J, Chambers AH, Barbey C, Bao Z, Plotto A, Bai J, et al. 2017. Identification of a methyltransferase catalyzing the final step of methyl anthranilate synthesis in cultivated strawberry. BMC Plant Biol. 17: 147.

5. Radulović NS, Miltojević AB, McDermott M, Waldren S, Parnell JA, Pinheiro MMG, et al. 2011. Identification of a new antinociceptive alkaloid $N$-methylantranilate from the essential oil of Choisya ternate Kunth. J. Ethnopharmacol. 135: 610-619.

6. Moio L, Etievant PX. 1995. Ethyl anthranilate, ethyl cinnamate, 2,3-dihydrocinnamate, and methyl anthranilate: four important odorants Identified in Binot Noir Wines of Burgundy. Am. J. Enol. Viticult. 46: 392-398.

7. Chambers AH, Evans SA, Folta KM. 2013. Methyl anthranilate and $\gamma$-decalactone inhibit strawberry pathogen growth and achene germination. J. Agri. Food Chem. 61: 12625-12633.

8. Clark L, Shah PS, Mason JR. 1991. Chemical repellency in birds: relationship between chemical structure and avoidance response. J. Exp. Zool. 260: 310-322.

9. Radulović NS, Miltojević AB, Randjelović PJ, Stojanović NM, Boylan F. 2013. Effects of methyl and isopropyl Nmethylanthranilates from Choisya ternata Kunth (Rutaceae) on experimental anxiety and depression in mice. Phytother. Res. 27: 1334-1338.
10. Correa E, Quiñones W, Echeverri F. 2016. Methyl-Nmethylanthranilate, a pungent compound from Citrus reticulata Blanco leaves. Pharm. Biol. 54: 569-571.

11. Fuleki, T. 1972. Changes in the chemical composition of Concord grapes grown in Ontario during ripening in the 1970 season. Can. J. Plant Sci. 52: 863-868.

12. Avery ML, Decker DG, Humphrey JS, Aronov E, Linscombe SD, Way MO. 1995. Methyl anthranilate as a rice seed treatment to deter birds. J. Wildl. Manag. 59: 50-56.

13. Cummings JL, Avery ML, Pochop PA, Davis Jr. JE, Decker DG, Krupa HW, et al. 1995. Evaluation of a methyl anthranilate formulation for reducing bird damage to blueberries. Crop. Prot. 14: 257-259.

14. Rohde B, Hans J, Martens S, Baumert A, Hunziker P, Matern U. 2008. Anthranilate $N$-methyltransferase, a branchpoint enzyme of acridone biosynthesis. Plant J. 53: 541-553.

15. Wang J, De Luca V. 2005. The biosynthesis and regulation of biosynthesis of Concord grape fruit esters, including 'foxy' methylanthranilate. Plant J. 44: 606-619.

16. Coleman JP, Hudson LL, McKnight SL, Farrow JM, Calfee MW, Lindsey CA, et al. 2008. Pseudomonas aeruginosa PqsA is an anthranilate-coenzyme A ligase. J. Bacteriol. 190: 1247-1255.

17. Jeandet P, Vasserot Y, Chastang T, Courot E. 2013. Engineering microbial cells for the biosynthesis of natural compounds of pharmaceutical significance. BioMed Res. Int. 2013: 13 .

18. Song MK, Lee SJ, Kang YY, Lee Y, Mok H, Ahn J-H. 2017. Biological synthesis and anti-inflammatory activity of arylalkylamine. Appl. Biol. Chem. 60: 597-602.

19. Lee SJ, Sim GY, Kang H, Yeo WS, Kim B-G, Ahn J-H. 2018. Synthesis of avenanthramides using engineered Escherichia coli. Microb. Cell Fact. 17: 46.

20. Choo HJ, Kim EJ, Kim SY, Lee Y, Kim B-G, Ahn J-H. 2018. Microbial synthesis of hydroxytyrosol and hydroxysalidroside. Appl. Biol. Chem. 61: 295-301.

21. Kim M, Kim H, Lee W, Lee Y, Kwon SW, Lee J. 2015. Quantitative shotgun proteomics analysis of rice anther proteins after exposure to high temperature. Int. J. Genomics 2015: 238704.

22. Pittard AJ, Davidson BE. 1991. TyrR protein of Escherichia coli and its role as repressor and activator. Mol. Microbiol. 5: 1585-1592.

23. Shoeman R, Redfield B, Coleman T, Greene RC, Smith AA, Brot N, et al. 1985. Regulation of methionine synthesis in Escherichia coli: effect of metj gene product and $S$ adenosylmethionine on the expression of the metF gene. Proc. Natl. Acad. Sci. USA 82: 3601-3605. 